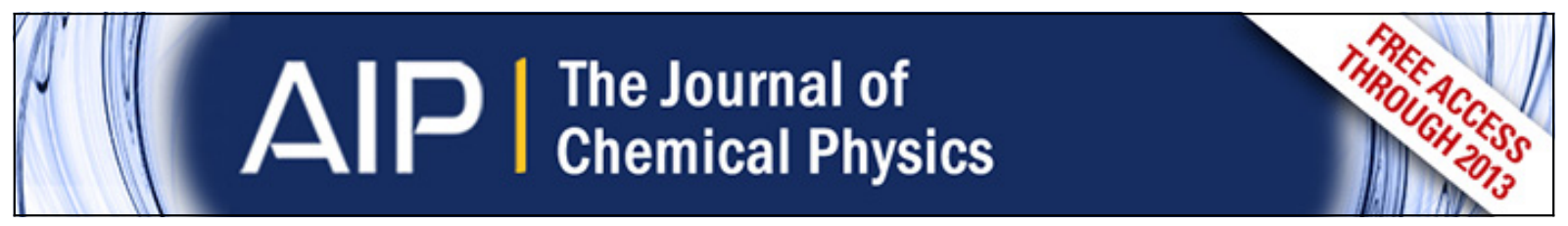

Shock response of single crystal and nanocrystalline pentaerythritol tetranitrate: Implications to hotspot formation in energetic materials

Y. Cai, F. P. Zhao, Q. An, H. A. Wu, W. A. Goddard III, and S. N. Luo

Citation: The Journal of Chemical Physics 139, 164704 (2013); doi: 10.1063/1.4825400

View online: http://dx.doi.org/10.1063/1.4825400

View Table of Contents: http://scitation.aip.org/content/aip/journal/jcp/139/16?ver=pdfcov

Published by the AIP Publishing

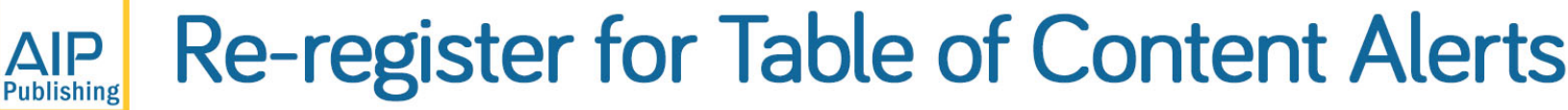

\section{Create a profile.}




\title{
Shock response of single crystal and nanocrystalline pentaerythritol tetranitrate: Implications to hotspot formation in energetic materials
}

\author{
Y. Cai, ${ }^{1,2}$ F. P. Zhao, ${ }^{1,2}$ Q. An, ${ }^{3}$ H. A. Wu, ${ }^{1, \text { a) }}$ W. A. Goddard III, ${ }^{3}$ and S. N. Luo, ${ }^{2, b)}$ \\ ${ }^{1}$ CAS Key Laboratory of Materials Behavior and Design, Department of Modern Mechanics, \\ University of Science and Technology of China, Hefei, Anhui 230027, People's Republic of China \\ ${ }^{2}$ The Peac Institute of Multiscale Sciences and Sichuan University, Chengdu, Sichuan 610207, \\ People's Republic of China \\ ${ }^{3}$ Materials and Process Simulation Center, California Institute of Technology, Pasadena, \\ California 91125, USA
}

(Received 9 August 2013; accepted 3 October 2013; published online 23 October 2013)

\begin{abstract}
We investigate shock response of single crystal and nanocrystalline pentaerythritol tetranitrate (PETN) with a coarse-grained model and molecular dynamics simulations, as regards mechanical hotspot formation in the absence or presence of grain boundaries (GBs). Single crystals with different orientations, and columnar nanocrystalline PETN with regular hexagonal, irregular hexagonal, and random GB patterns, are subjected to shock loading at different shock strengths. In single crystals, shock-induced plasticity is consistent with resolved shear stress calculations and the steric hindrance model, and this deformation leads to local heating. For regular-shaped hexagonal columnar nanocrystalline PETN, different misorientation angles lead to activation of different/same slip systems, different deformation in individual grains and as a whole, different GB friction, different temperature distributions, and then, different hotspot characteristics. Compared to their regular-shaped hexagonal counterpart, nanocrystalline PETN with irregular hexagonal GB pattern and that with random GBs, show deformation and hotspot features specific to their GBs. Driven by stress concentration, hotspot formation is directly related to GB friction and GB-initiated crystal plasticity, and the exact deformation is dictated by grain orientations and resolved shear stresses. GB friction alone can induce hotspots, but the hotspot temperature can be enhanced if it is coupled with GB-initiated crystal plasticity, and the slip of GB atoms has components out of the GB plane. The magnitude of shearing can correlate well with temperature, but the slip direction of GB atoms relative to GBs may play a critical role. Wave propagation through varying microstructure may also induce differences in stress states (e.g., stress concentrations) and loading rates, and thus, local temperature rise. GBrelated friction and plasticity induce local heating or mechanical hotspots, which could be precursors to chemical hotspot formation related to initiation in energetic materials, in the absence of other, likely more effective, means for hotspot formation such as void collapse. () 2013 AIP Publishing LLC. [http://dx.doi.org/10.1063/1.4825400]
\end{abstract}

\section{INTRODUCTION}

To initiate chemical reactions in an energetic material, sufficient energy must be deposited for bond breaking. Bowden and Yoffe ${ }^{1}$ first showed that the bulk heating due to mechanical impact was inadequate, and proposed that above-average heating in small regions, termed as "hotspots," provided sufficient energy density to start reaction locally. Hotspots are expected to play a key role in single crystal and polycrystalline explosives, polymer-bonded explosives, and liquid explosives. The sensitivity of an explosive is closely related to its response to an applied dynamic or thermal stress, presumably manifested through the productions of hotspots, and this response is strongly affected by material heterogeneities such as crystal defects or interfaces. ${ }^{2-4}$ Under dynamic loading, such heterogeneities can result in high heat or stress concentrations, i.e., hotspots, facilitating initiation and detonation of energetic materials. ${ }^{5-10}$

\footnotetext{
a)wuha@ustc.edu.cn

b) sluo@pims.ac.cn
}

Theoretical or simulation studies on condensed-phase energetic materials employ such techniques as static electronic structure, ${ }^{11-14}$ molecular dynamics (MD) simulations using empirical or electronic-structure-based force fields, ${ }^{15-17}$ as well as reactive force fields (ReaxFF), ${ }^{18,19}$ and ones based on coarse-grained (CG), particle-based methods. ${ }^{20-23}$ The energetic materials explored include nitromethane, ${ }^{11,15,16,24-29}$ octahydro-1,3,5,7-tetranitro-1,3,5,7-tetrazocine (HMX), ${ }^{30-34}$ hexa-hydro-1,3,5-trinitro-1,3,5-triazine (RDX), ${ }^{18,35-42} 1,3,5$ triamino-2,4,6-trinitrobenzene (TATB), ${ }^{12,13}$ and pentaerythritol tetranitrate (PETN), ${ }^{23}$ among others. ${ }^{14}$ See Rice and Sewell ${ }^{43}$ for a recent review on this subject.

PETN is of particular interest for its relative simplicity. Dick and co-workers ${ }^{44-47}$ performed extensive studies on single crystal PETN shocked along different orientations, and observed anisotropy in the threshold shock strength for initiation of detonation. PETN is sensitive to shocks impinged on (110) and (001) planes but relatively insensitive to on any other planes studied. ${ }^{45}$ Dick $^{46}$ proposed the steric hindrance model to explain this anisotropy. The steric hindrance model 
states that, for insensitive shock orientations, the readily accessible slip systems relieve the shear stresses imparted by shock wave passage, whereas for sensitive orientations, the lack of available slip systems leads to hindered shear. The inability to relieve shear stresses gives rise to close intermolecular contacts that facilitate bond breaking and subsequent reactions.

Winey and Gupta ${ }^{48}$ developed an anisotropic continuum model to describe the thermomechanical response of unreacted PETN single crystals subjected to shock wave loading. Eason and Sewell ${ }^{49}$ performed MD simulations of shock waves in crystalline PETN using a nonreactive force field. They characterized the mechanical deformation processes for shocks along [100] and [001] directions. Zybin and coworkers ${ }^{19,50}$ used the ReaxFF reactive force field to determine the primary PETN reaction products resulting from shock compression ${ }^{19}$ and rapid compression and shear. ${ }^{50}$ They determined that $\mathrm{NO}_{2}$ is the primary reaction that occurs following strong shock loading along the [100] direction. ${ }^{19}$ In the compress-and-shear simulations, they observed a higher temperature and stress jump for the sensitive orientations, attributed to the close intermolecular contacts. ${ }^{50}$

Grain boundaries (GBs) are ubiquitous in crystalline energetic materials and may play an important role in hotspot formation. Nonetheless, their role in the thermal-mechanicalchemical response of shocked energetic materials has never been investigated. In this article, we report MD simulations of shock response of single crystal and nanocrystalline PETN using a CG model, which reduces computational cost while retaining reasonable accuracy ${ }^{20}$ necessary for simulations of large polycrystalline systems. Recently, Arman et al. ${ }^{51}$ applied a CG-MD shock simulations to polymers, and Izvekov et al. ${ }^{23}$ performed multiscale CG simulations on RDX. Our objective is to characterize in atomic detail the mechanical deformation at GBs and in grain interiors, and make connections to hotspot formation. These "mechanical" hotspots may be the initiation zones of chemical reactions, leading to "chemical" hotspots and then detonation. "Chemical" hotspots cannot be simulated with current CG model, and reactive force fields are necessary for this purpose. We find that GBs enhance greatly the formation of mechanical hotspots via GB friction and GBinitiated crystal plasticity, suggesting GB-related deformation as a plausible mechanism for hotspot formation in energetic materials. Section II addresses the methodology of CG-MD simulations and data analysis, followed by results and discussion in Sec. III. Conclusions are presented in Sec. IV.

\section{METHODOLOGY}

We use the Large-scale Atomic/Molecular Massively Parallel Simulator (LAMMPS), ${ }^{52}$ and a CG model by Gee et al. $^{20}$ in our MD simulations. Figures 1 (a) and 1(b) display the molecular structure of PETN and corresponding CG representation, as well as the CG unit cell. The crystal structure is body-centered tetragonal; the lattice parameters are $a=b=9.38 \AA, c=6.7 \AA$, and $\alpha=\beta=\gamma=90^{\circ}$. In the CG model, ${ }^{20}$ each $\mathrm{NO}_{2}$ group in a PETN molecule is lumped into a pendent " $\mathrm{B}$ " bead, and the remaining atoms are grouped into a central "A" bead. For the interactions be-
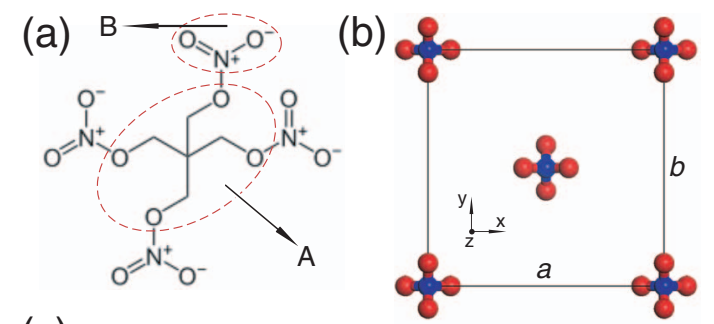

(c)

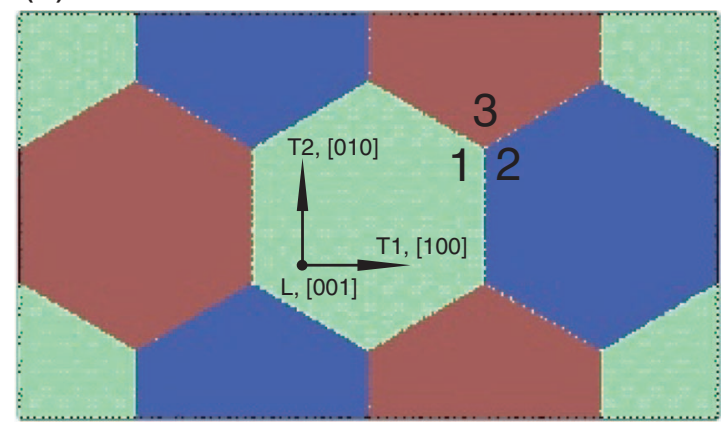

FIG. 1. Molecular, crystal, and nanocrystalline structures of PETN. (a) Molecular structure of PETN, and its CG representation with "A" and "B" beads. (b) CG-model unit cell viewed along [001]. (c) Hexagonal columnar nanocrystalline configuration of PETN containing three types of grains (1-3). Color coding denotes crystal orientation.

tween the coarse-grained beads, we consider only the intramolecular $\mathrm{A}-\mathrm{B}$ bond interaction, described with the harmonic form $E_{\text {bond }}\left(r_{\mathrm{AB}}\right)=K_{\text {bond }}\left(r_{\mathrm{AB}}-b_{\mathrm{AB}}^{0}\right)^{2}$; and the $\mathrm{B}-\mathrm{B}$ nonbond, van der Waals interaction in 12-6 Lennard-Jones form, $E_{\mathrm{vdW}}\left(r_{\mathrm{BB}}\right)=\varepsilon_{\mathrm{BB}}\left[\left(\sigma_{\mathrm{BB}} / r_{\mathrm{BB}}\right)^{12}-2\left(\sigma_{\mathrm{BB}} / r_{\mathrm{BB}}\right)^{6}\right] .{ }^{20}$ Here, $r$ denotes the distance between two beads. In our simulations, $\varepsilon_{\mathrm{BB}}=1.38 \mathrm{kcal} / \mathrm{mol}$ and $\sigma_{\mathrm{BB}}=4.81 \AA$. The bond interaction parameters $K_{\mathrm{bond}}$ and $b_{\mathrm{AB}}^{0}$ are assigned generic values of $20.0 \mathrm{kcal} / \mathrm{mol}$ and $2.6 \AA$, respectively. All other interactions, including the $\mathrm{B}-\mathrm{A}-\mathrm{B}$ angle term, and intermolecular $\mathrm{A}-\mathrm{A}$ and $\mathrm{A}-\mathrm{B}$ interactions, are neglected. The effective masses for $A$ and $B$ beads in atomic mass units are 132.12 and 46.0052, respectively. ${ }^{20}$ The accuracy of the CG model for PETN was examined in Ref. 20, including hydrostatic compression to $10 \mathrm{GPa}$. The slip systems as resolved from our shock simulations are also consistent with independent studies with allatom MD simulations, experiments, steric hinderance model, and continuum model (see discussion below). Despite its simplicity, this CG model is of reasonable accuracy for plastic deformation.

Our PETN shock simulations employ columnar nanocrystalline configurations. They are chosen for the convenience of structure analysis and visualization, circumventing the complexities of three-dimensional (3D) random GBs without compromising our insights into the underlying physics. The nanocrystals are constructed with the Voronoi method. ${ }^{53}$ In this method, a set of grain centers is first specified, and for each center there is a corresponding region consisting of all atoms closer to that center than to any other centers. The formal definition is

$$
\mathcal{G}_{i}=\left\{\mathcal{A} \in \mathcal{S} \mid d\left(\mathcal{A}, C_{i}\right) \leq d\left(\mathcal{A}, C_{j}\right) \text { for all } j \neq i\right\} .
$$

Here, $\mathcal{S}$ is a space created with a distance function $d . \mathcal{A}, \mathcal{G}_{i}$, and $C_{i}$ denote atoms, grain $i$, and grain center $i$, respectively. 
GBs may form polygons of different shapes due to different grain centers chosen, and different number of grains may be resulted.

A unit columnar nanocrystalline configuration with regular-shaped hexagonal grains and tilt GBs is shown in Fig. 1(c). The rotation axis is [001], and type-1 grains are the reference grains. Type- 2 and 3 grains are rotated along the $L$ axis (i.e., [001]) by $-\theta$ and $\theta$ relative to type- 1 grains, respectively. Here, $\theta$ is the rotation angle or angular misorientation. The cross section is about $100 \mathrm{~nm} \times 60 \mathrm{~nm} \times 4 \mathrm{~nm}$, respectively, along the $T 1-, T 2-$, and $L$-directions [Fig. 1(c)]. The $T 1-/ T 2$-direction is essentially the zigzag/armchair direction in a honeycomb structure. Different shock orientations (the $T 1-, T 2$-, and $L$-directions) and angular misorientations are explored in our simulations. The unit configurations are replicated along the shock directions. The dimensions along the shock direction are about $200 \mathrm{~nm}$ (800 000 atoms), $240 \mathrm{~nm}$ (1 600000 atoms), and $100 \mathrm{~nm}$ (10 000000 atoms), respectively, for the $T 1$-, $T 2$-, and $L$-direction shocks. We also examine shock response of single crystals, and the configurations are about $350 \mathrm{~nm} \times 20 \mathrm{~nm} \times 20 \mathrm{~nm}(\sim 2000000$ atoms $)$. The resulting configurations are annealed, relaxed, and finally thermalized at the ambient conditions with the constantpressure-temperature ensemble and 3D periodic boundary conditions.

Shock waves are driven by a rigid piston from the left, which is accelerated from 0 to a terminal velocity $u_{\mathrm{p}}$ within $10 \mathrm{ps}$, and then stays at this velocity. The shock simulations are performed with the microcanonical ensemble. The shock loading direction is denoted as the $x$-axis, and the other two orthogonal axes, the $y$-and $z$-axes. In the single crystal cases, the crystallographic plane normal to the shock direction is referred to as the shock plane for simplicity. Periodic boundary conditions are applied only along the $y$-and $z$-axes. The time step for integration of the equation of motion is $1 \mathrm{fs}$, and the run durations are up to $100 \mathrm{ps}$.

We perform 1D and 2D binning analyses ${ }^{54,55}$ to resolve spatially such physical properties as density $(\rho)$, stress tensor $\left(\sigma_{i j}\right)$, particle velocity $(\mathbf{u})$, and temperature $(T)$ profiles at different stages of compression. In the cases of columnar nanocrystalline PETN, averaging along the $z$-axis or [001] is applied for $2 \mathrm{D}$ analysis. The bin size is about $8 \AA$. To calculate $T$ and $\sigma_{i j}$ within each bin, we need to remove its center-of-mass velocity, $\bar{v}_{i}(i=x, y$, and $z)$, or apply corrections: $\quad \Delta T=-\left(m / 3 k_{\mathrm{B}}\right)\left(\bar{v}_{x}^{2}+\bar{v}_{y}^{2}+\bar{v}_{z}^{2}\right), \quad$ and $\Delta \sigma_{i j}$ $=-(N m / V) \bar{v}_{i} \bar{v}_{j}$, where $m$ is the atomic mass, $V$ is the bin volume, and $N$ is the number of atoms in the volume under consideration.

The atomic-level deformation can be characterized with the slip vector ${ }^{56,57}$

$$
\mathbf{s}_{i}=-\frac{1}{n_{\mathbf{s}}} \sum_{i \neq j}^{n}\left(\mathbf{x}_{i j}-\mathbf{X}_{i j}\right) .
$$

Here, $n$ is the number of the nearest neighbors to atom $i, n_{\mathbf{s}}$ is the number of the slipped neighbors $j$, and $\mathbf{x}_{i j}$ and $\mathbf{X}_{i j}$ denote the vector (between atom $i$ and $j$ ) difference in current and reference configurations, respectively. The reference configurations are the preshock structures. Similarly, the maximum relative displacement (MRD) is defined as

$$
\mathbf{s}_{i}=\mathbf{x}_{i j}-\mathbf{X}_{i j}:\left|\mathbf{x}_{i j}-\mathbf{X}_{i j}\right|_{\max } .
$$

The latter definition is used in our analysis, and the scalar slip is $s_{i}=\left|\mathbf{s}_{i}\right|$. When calculating MRD, we consider only A-type beads.

We use resolved shear stress (RSS) for discussing plastic deformation mechanisms. RSS is defined as the component of shear stress, resolved in the direction of slip on the corresponding slip plane. RSS reflects the driving force for shear deformation. The full stress tensor in a selected region (e.g., the supported shock region) is calculated via averaging all the bins in this region, for a given frame; we then find or choose a relevant slip system, and project this stress tensor onto the slip plane along the slip direction to obtain RSS. ${ }^{58}$

\section{RESULTS AND DISCUSSION}

Shock loading is applied at several piston velocities. We explore $u_{\mathrm{p}}=0.3 \mathrm{~km} / \mathrm{s}-2.0 \mathrm{~km} / \mathrm{s}$ for single crystal PETN, and two representative piston velocities, $1.0 \mathrm{~km} / \mathrm{s}$ and $1.5 \mathrm{~km} / \mathrm{s}$, for columnar nanocrystalline PETN. We perform $1 \mathrm{D}$ and $2 \mathrm{D}$ binning analyses as well as deformation analysis, and examine the shock equation of state, wave propagation, slip systems in single crystals, plastic deformation, hotspots, and the microstructure effects including GB characteristics and anisotropy. We present the results in Figs. 2-15 and Table I, along with discussions where appropriate.

\section{A. Single crystal PETN}

We first discuss single crystals, since they are constituents of a polycrystalline solid, and their slip systems are particularly relevant. They can also be used for checking the accuracy of the CG model. Figure 2 shows the shock velocity-particle velocity $\left(u_{\mathrm{s}}-u_{\mathrm{p}}\right)$ relations of single crystal PETN for five low-index shock planes: (100), (110), (001), (101), and (111), including MD simulation and experimental results. ${ }^{59,60}$

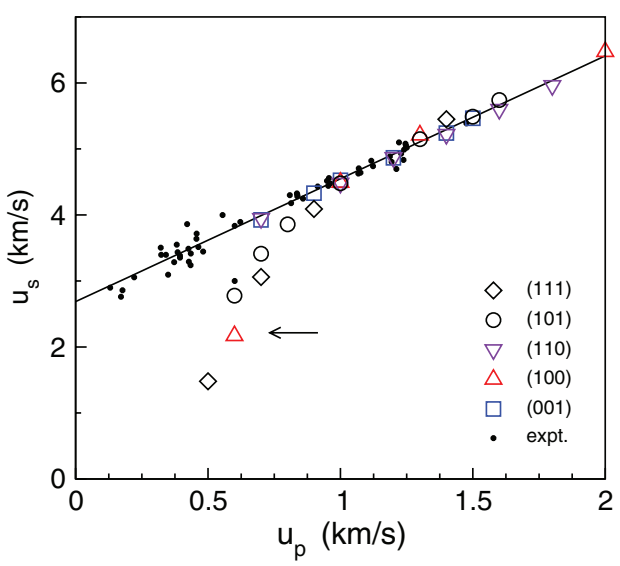

FIG. 2. Shock velocity $\left(u_{\mathrm{s}}\right)$ vs. particle velocity $\left(u_{\mathrm{p}}\right)$ for single crystal PETN obtained from MD simulations (open symbols) and experiments (dots, Refs. 59-61). The line represents the linear fitting to the experimental results: $u_{\mathrm{s}}=2.69+1.86 u_{\mathrm{p}}$ in $\mathrm{km} / \mathrm{s}$. Only the plastic wave speeds are plotted for clarity. 


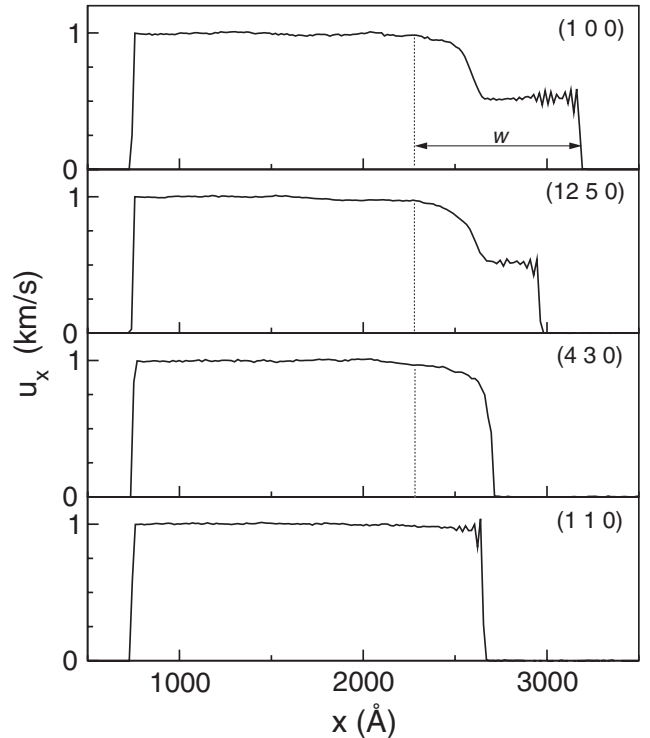

FIG. 3. 1D $u_{x}-x$ profiles of single crystal PETN for different shock loading planes as indicated. $u_{\mathrm{p}}=1.0 \mathrm{~km} / \mathrm{s}$ and $t=60 \mathrm{ps}$. $w$ indicates an effective "shock rise" width. Shock direction: left $\rightarrow$ right.

The Hugoniot states, where the system has achieved thermal and mechanical equilibria within MD time scales, are obtained from the plateau regions in the $u_{x}(x)$ profiles (e.g., Fig. 3) and Hugoniot jump conditions. PETN single crystals show pronounced anisotropy in elasticity and plasticity in terms of wave speeds, Hugoniot elastic limit (HEL), and features in the $u_{\mathrm{s}}-u_{\mathrm{p}}$ plots. For the (100), (101), and (111)

(a)

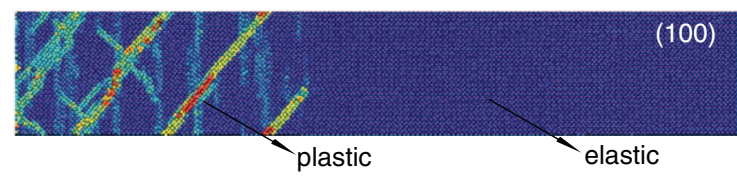

(b)

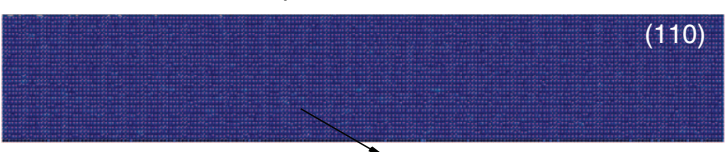

(c)

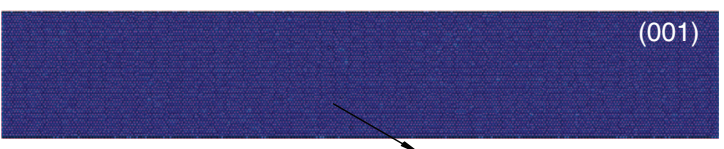

(d)

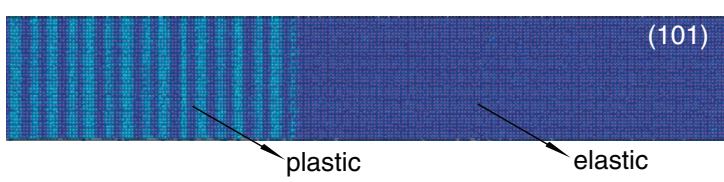

(f)

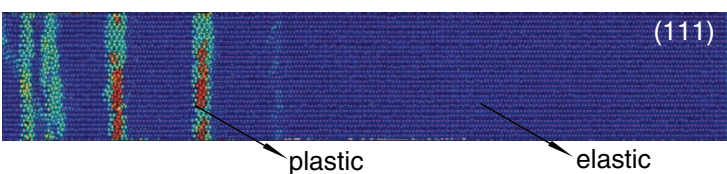

shock direction

FIG. 4. (a)-(f) Snapshots showing pure elastic or elastic/plastic deformation in single crystal PETN shock-loaded at $u_{\mathrm{p}}=0.5 \mathrm{~km} / \mathrm{s}$ along different crystal orientations. The crystallographic planes normal to the shock directions (shock planes) are noted. Color coding refers to MRD in $\AA$.
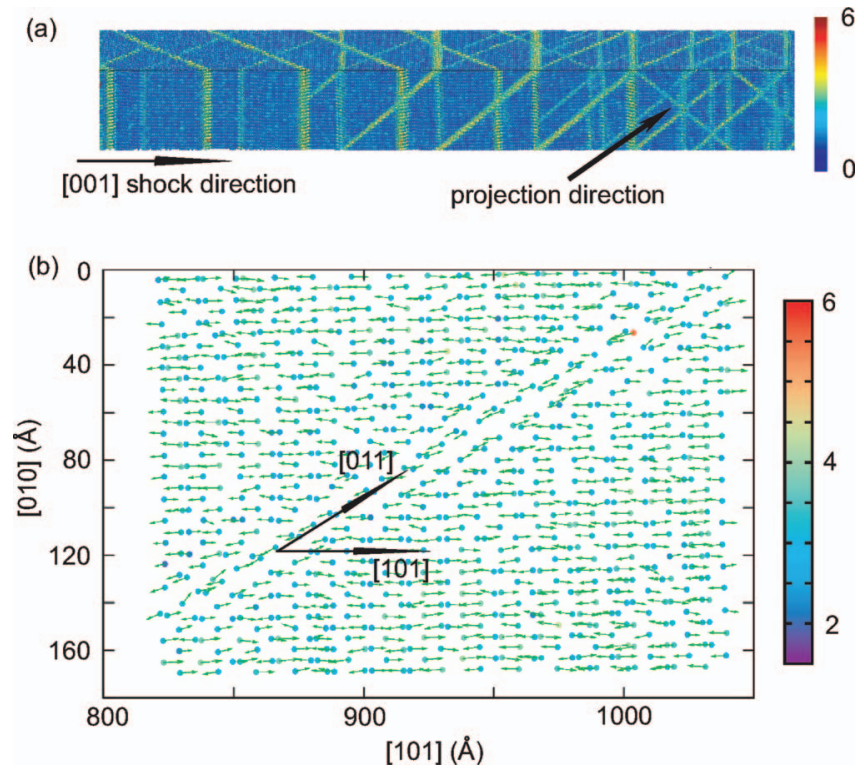

FIG. 5. (a) Snapshot of single crystal PETN shock-loaded at $u_{\mathrm{p}}=0.6 \mathrm{~km} / \mathrm{s}$ along [001]. (b) Slip vector plot on a selected plane as indicated by the arrow in (a). The slip directions for two slip systems are noted. Color coding refers to MRD in $\AA$.

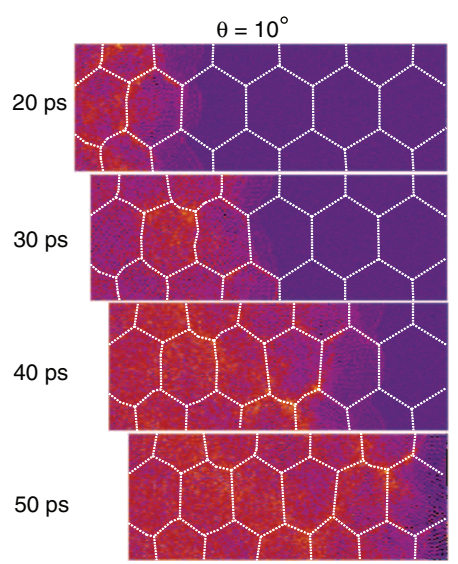

(1)

20 ps

$\theta=30^{\circ}$

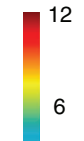

6 0

$30 \mathrm{ps}$
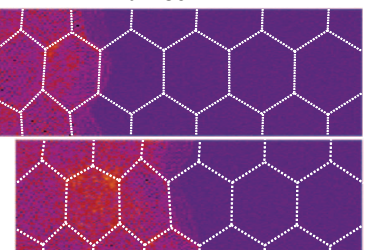

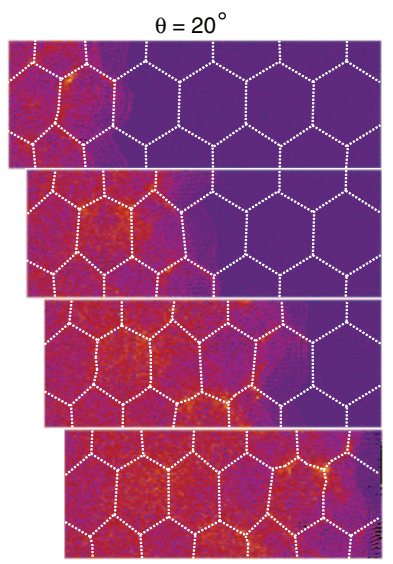

$40 \mathrm{ps}$

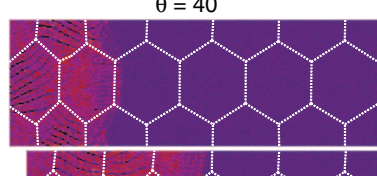

$50 \mathrm{ps}$
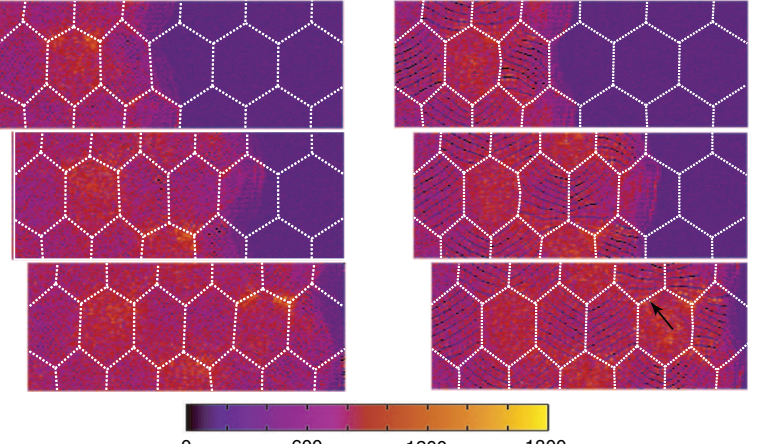

$1200 \quad 1800$

FIG. 6. Evolution of 2D distributions of temperature ( $T$; in $\mathrm{K})$ in regular hexagonal columnar nanocrystalline PETN, averaged over the $L$-direction for the $T 1$-direction loading with $u_{\mathrm{p}}=1.0 \mathrm{~km} / \mathrm{s}$, for different angular misorientations $(\theta)$. The white dotted lines indicate the approximate locations of GBs. Shock direction: left $\rightarrow$ right. 


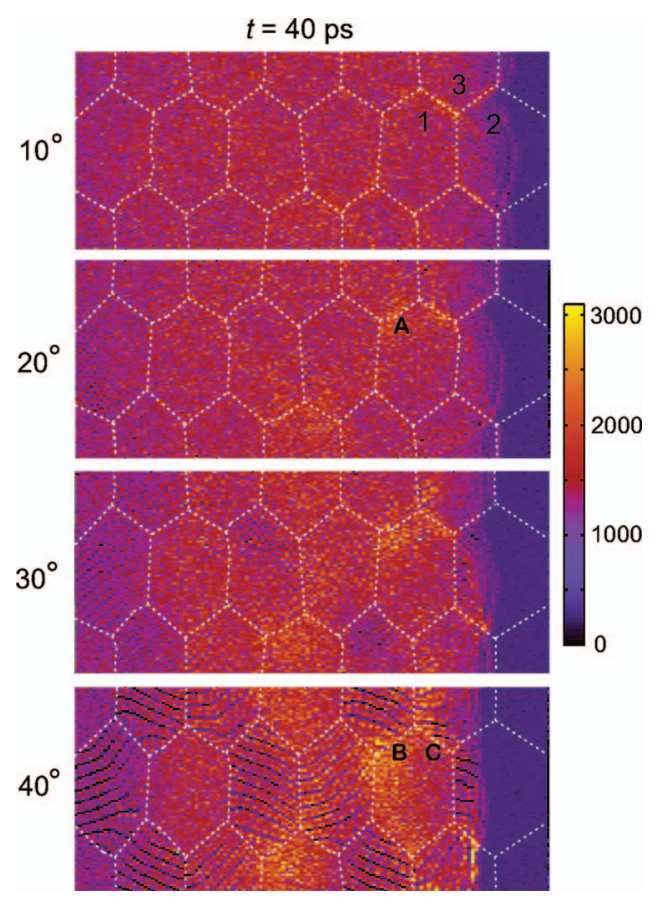

FIG. 7. 2D distribution of temperature in regular hexagonal columnar nanocrystalline PETN with different $\theta$, averaged over the $L$-direction for the T1-direction loading at $u_{\mathrm{p}}=1.5 \mathrm{~km} / \mathrm{s}$. The white dotted lines indicate the approximate locations of grain boundaries. Shock direction: left $\rightarrow$ right.
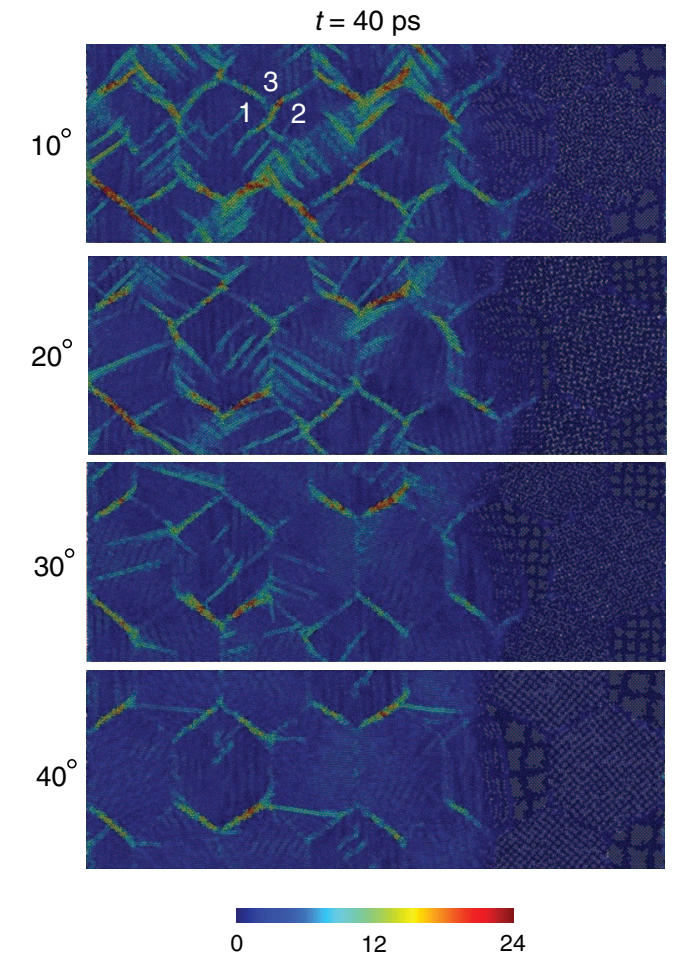

FIG. 8. 2D distribution of MRD in regular hexagonal columnar nanocrystalline PETN with different $\theta$, for the $T 1$-direction loading at $u_{\mathrm{p}}=1.0 \mathrm{~km} / \mathrm{s}$, for different angular misorientations $(\theta)$. Shock direction: left $\rightarrow$ right. MRD is in $\AA$. (a)

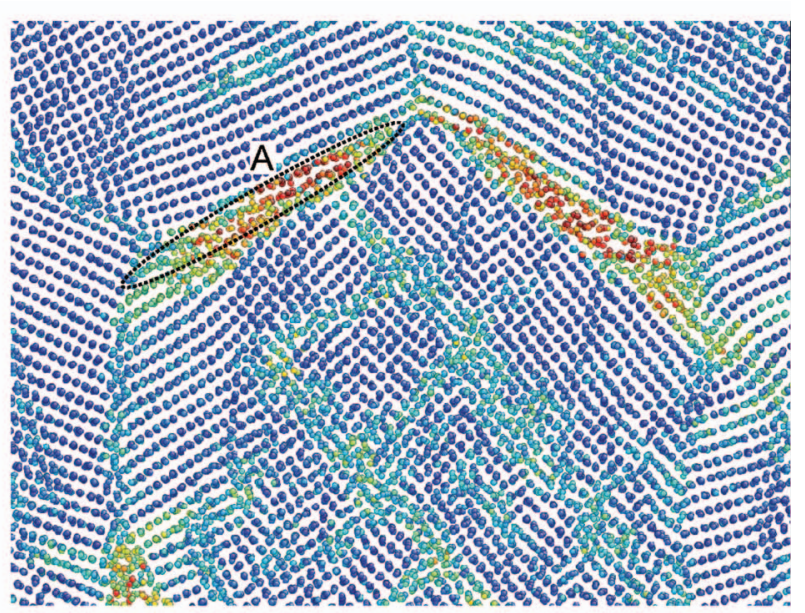

(b)

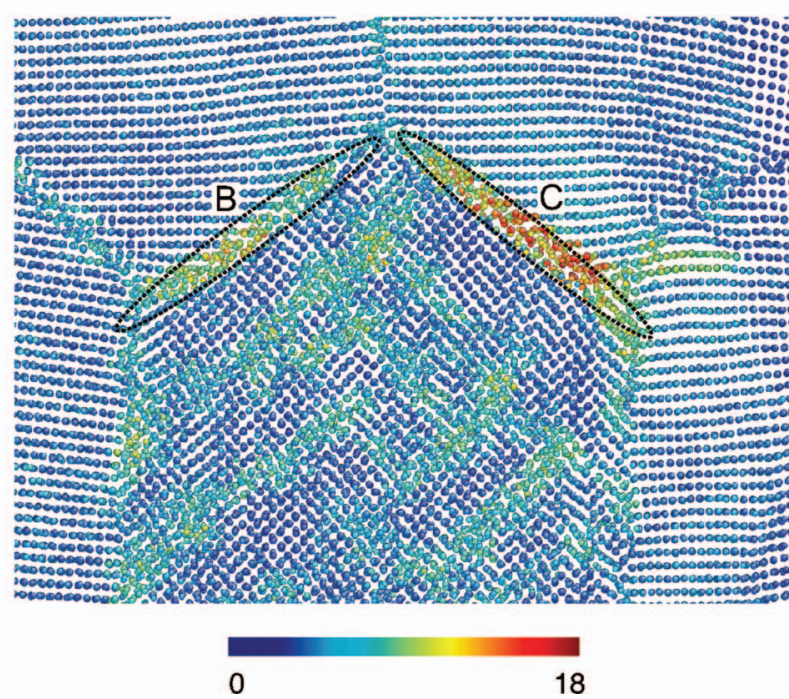

FIG. 9. Atomic configurations of regular hexagonal columnar nanocrystalline PETN for the T1-direction loading with $u_{\mathrm{p}}=1.5 \mathrm{~km} / \mathrm{s}$, for $\theta=20^{\circ}$ (a) and $40^{\circ}$ (b). Shock direction: left $\rightarrow$ right. MRD is in $\AA$. GBs $A-C$ correspond to $A-C$ in Fig. 7 .

shocks, the elastic-plastic or two-wave structure is observed at the intermediate shock strengths, and the plastic wave overtakes the elastic precursor at high shock strengths (Figs. 2 and 3). Only the plastic wave speeds are plotted in Fig. 2 for clarity. However, the (110) and (001) shocks only show single-wave structures, likely due to the small difference in elastic-plastic wave speeds, and limited sample lengths which are insufficient for separating the elastic and plastic waves at MD time scales. In the plastic regime, the anisotropy as seen in the $u_{\mathrm{s}}-u_{\mathrm{p}}$ plot diminishes, indicating the isotropy of PETN when the plasticity is well achieved. The MD results are in accord with the experimental data ${ }^{59,60}$ in the plastic regime for high shock strengths, a manifestation of the high accuracy of the CG model for PETN. At low shock strengths, the plastic wave speeds from MD simulations are substantially lower than the experimental results for (111), (101), and (100). The deviation at lower shock strengths (indicated by the arrow in Fig. 2) for certain, not all, shock orientations is a common phenomenon in MD simulations of plastic shock waves in single crystals with certain orientations, including Lennard-Jonesium and metals described 

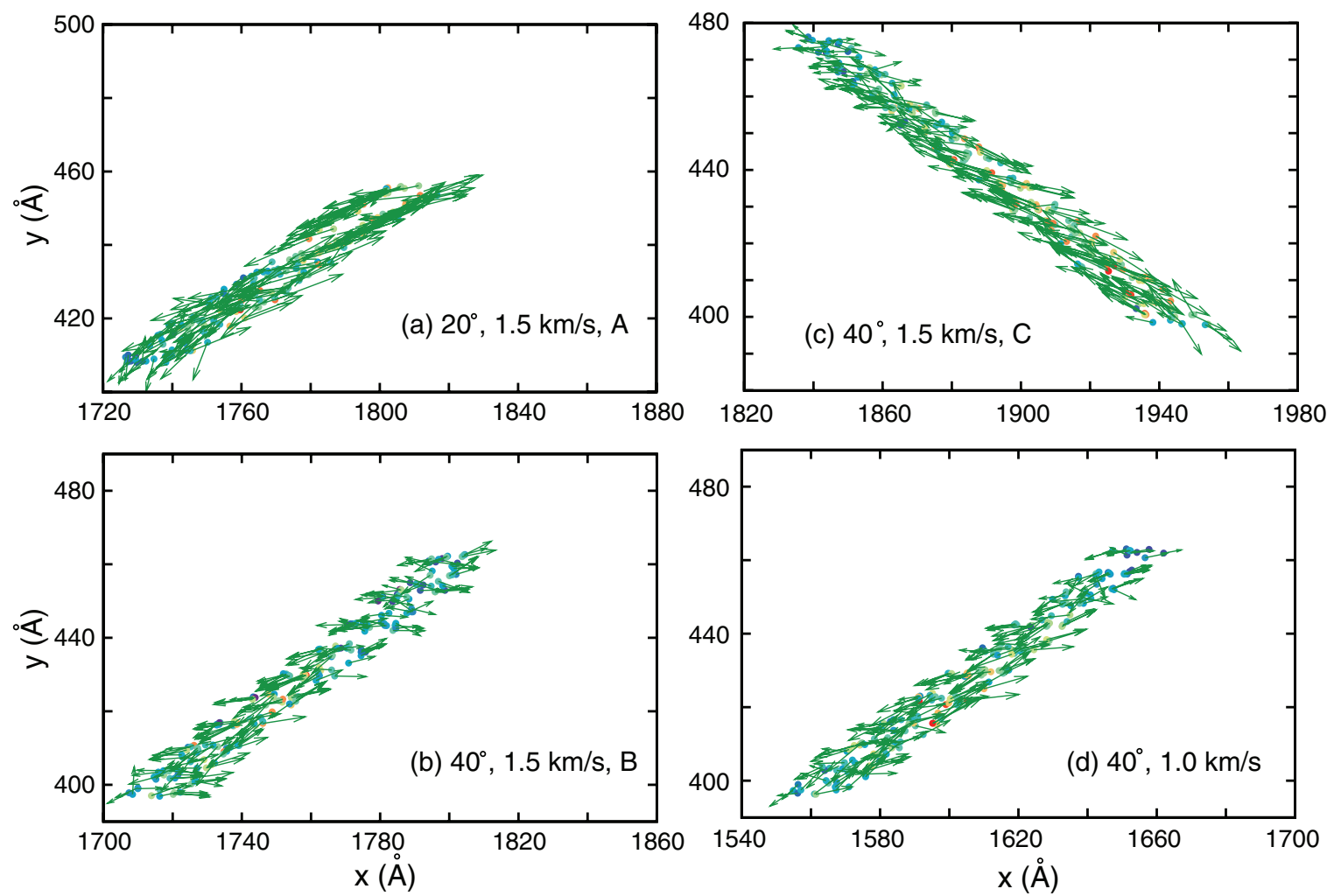

FIG. 10. Vector plots of MRD on selected grain boundaries in regular hexagonal columnar nanocrystalline PETN with different $\theta$, for the $T 1$-direction loading at different $u_{\mathrm{p}}$. Shock direction: left $\rightarrow$ right. MRD is in $\AA$. (a)-(c) correspond to $A-C$ in Fig. 9, respectively. (d) refers to the GB in Fig. 6 indicated by the arrow.
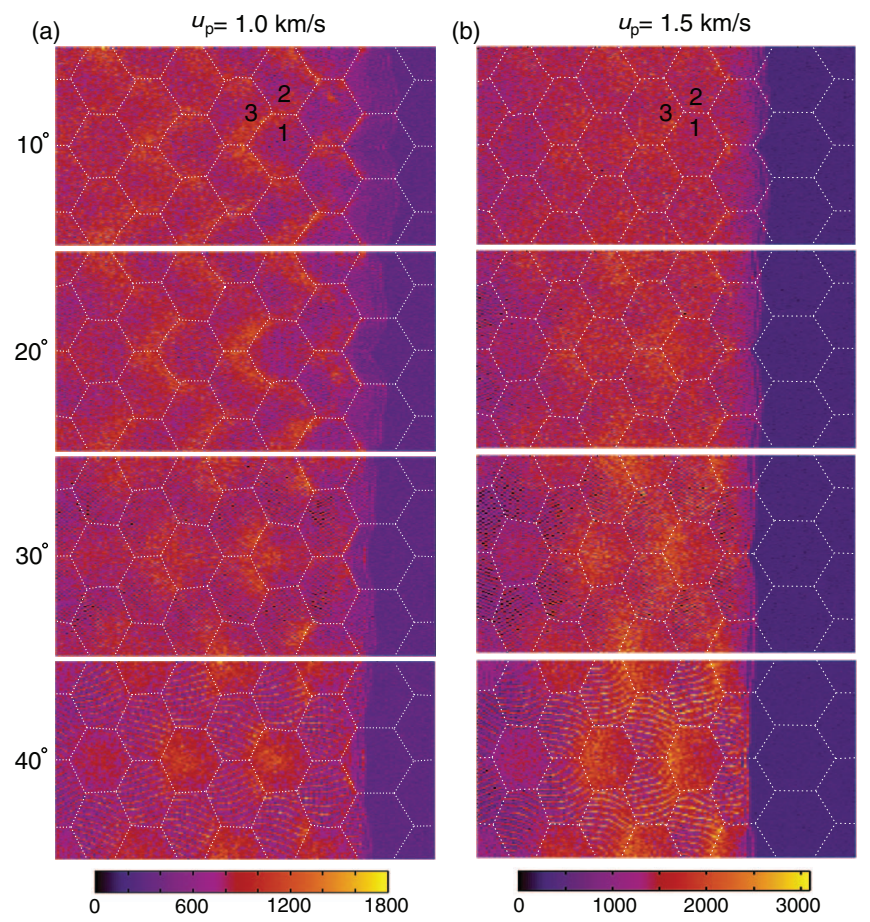

FIG. 11. 2D distribution of temperature in regular hexagonal columnar nanocrystalline PETN with different $\theta$, averaged over the $L$-direction for the $T 2$-direction loading at $u_{\mathrm{p}}=1.0 \mathrm{~km} / \mathrm{s}$ (a) and $1.5 \mathrm{~km} / \mathrm{s}$ (b). The white dotted lines indicate the approximate locations of grain boundaries. Shock direction: left $\rightarrow$ right. by accurate embedded-atom-method (EAM) potentials (e.g., Ta). ${ }^{62,63}$ This deviation is not due to the deficiencies in the force fields, but rather the unavoidably high strain rates (short time scale) in such simulations, which give rise to incomplete plasticity, underdeveloped plastic wave, and thus undervalued plastic wave strength and wave speed.

To examine the plastic deformation of PETN single crystals in more detail, we utilize MRD analysis at atomistic scales. Figure 4 shows snapshots for different shock planes $\left(u_{\mathrm{p}}=0.5 \mathrm{~km} / \mathrm{s}\right)$. For elastic deformation, MRD is negligible, while it increases substantially with increasing degree of plastic deformation. MRDs for (110) and (001) loading are around zero, indicating elastic deformation. By contrast, the MRD distributions display two distinct regions (elastic and plastic), and MRDs are about 3-12 $\AA$ in the plastic region. This result is consistent with expectations from the steric hindrance model. In our simulations, shocks on the "sensitive" (110) and (001) planes do not activate any slip systems, so little or no inelastic deformation occurs [Figs. 4(b) and 4(c)], and the strain energy from the shock is stored due to large "steric hindrance." To the contrary, shocks on the "insensitive" (100), (101), and (111) planes do activate slip systems with the shear stresses relieved, leading to pronounced inelastic deformation evident in Figs. 4(a), 4(d), and 4(e).

It is useful to identify the exact slip systems from MRD for different shock planes. We use the (001) shock as an example (Fig. 5). Figure 5(a) shows that at least three slip systems 


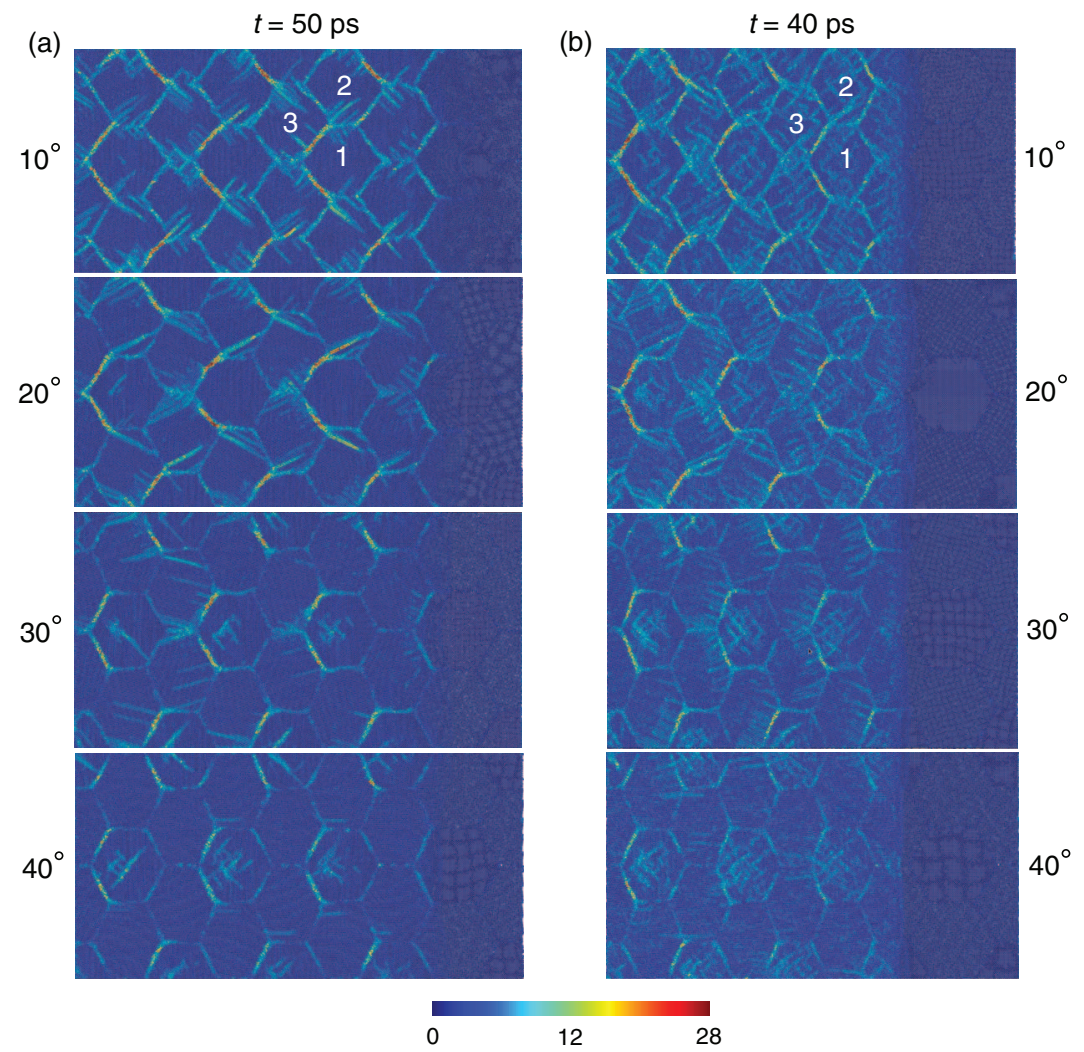

FIG. 12. 2D distribution of MRDs in regular hexagonal columnar nanocrystalline PETN with different $\theta$, for the $T 2$-direction loading at $u_{\mathrm{p}}=1.0 \mathrm{~km} / \mathrm{s}$ (a) and $1.5 \mathrm{~km} / \mathrm{s}$ (b). Shock direction: left $\rightarrow$ right.

are activated. We take a slip plane indicated by the arrow in Fig. 5(a), and plot the atomic configuration and slip vectors projected onto the slip plane [Fig. 5(b)]. The slip plane, (101), intersects with another slip plane, so two slip directions are identified, [011] and [101]. A slip system (101)[101] is then determined. The slip systems directly identified from our shock simulations for different shock planes are listed in Table I.

For each slip system, RSS or the driving force for shear deformation can be calculated. Shocks along different orientations may activate different or same slip systems, but highly likely, involve different RSS. For seven shock planes, we calculate the stress tensors of the shocked zone in a single crystal loaded at $u_{\mathrm{p}}=0.3 \mathrm{~km} / \mathrm{s}$, where no inelastic deformation occurs in all these shock planes. We then project the stress tensor onto different slip systems, either identified from our simulations or taken from literature, to obtain RSS. Table I lists the RSS results of selected slip systems for seven shock planes.

A higher RSS normally indicates more ease in activating a slip system (there are exceptions, though). For a given shock strength, the RSS values of the slip systems that have been observed in our simulations are relatively higher than those of other slip systems (Table I), since they are relatively easier to activate. The RSS for "insensitive" (100), (101), and (111) shock planes are significantly higher than those for "sensitive" (110) and (001) planes, i.e., it is easier to activate the slip systems for "insensitive" shock planes than "sensitive" planes. The variations in RSS for different shock planes also explain the anisotropy observed in Fig. 4. The RSS values for (12 50 ) and (4 30 ) shock planes, along with other planes, allow us to see the trend in RSS variations with the angular misorientation, by which a single crystal is rotated around [001] (relative to grain 1 in Fig. 1(c); see discussion in Sec. III B).

The slip systems were discussed previously in independent studies, including indirect experiments, all-atoms MD simulations, and continuum-level models. ${ }^{44,46,48-50}$ Although not a direct comparison, our simulations are consistent with these studies. The anisotropy of shock response in singlecrystal PETN revealed by our simulations is consistent with the steric hindrance model which was developed by Dick et $a .^{46}$ and demonstrated in following studies, including molecular mechanics simulations, MD simulations with allatom model or ReaxFF, and simulations with continuum-level models. $^{48-50}$

Plastic deformation nucleates homogeneously in a single crystal upon shock loading. A relevant question is whether such homogeneous nucleation of plasticity can induce mechanical hotspots that lead to chemical spots. It is highly possible since crystal plasticity heating is the most plausible, and possibly the only, means in a shocked single crystal without other defects.

\section{B. Regular hexagonal columnar nanocrystalline PETN shocked along the $T 1$-direction}

Shock simulations are performed of columnar nanocrystalline PETN with regular-hexagon-shaped grains and 
(a)
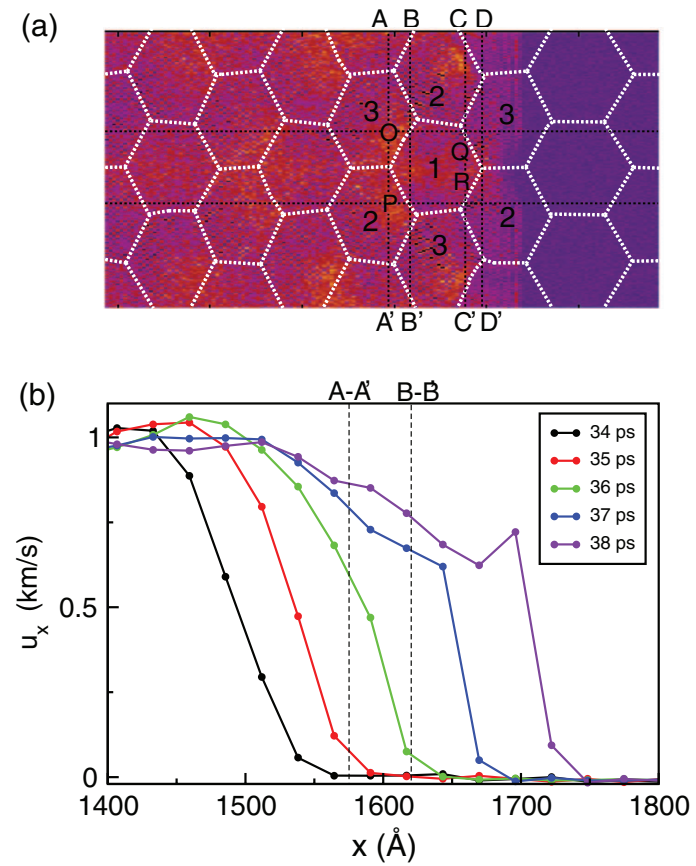

(c)

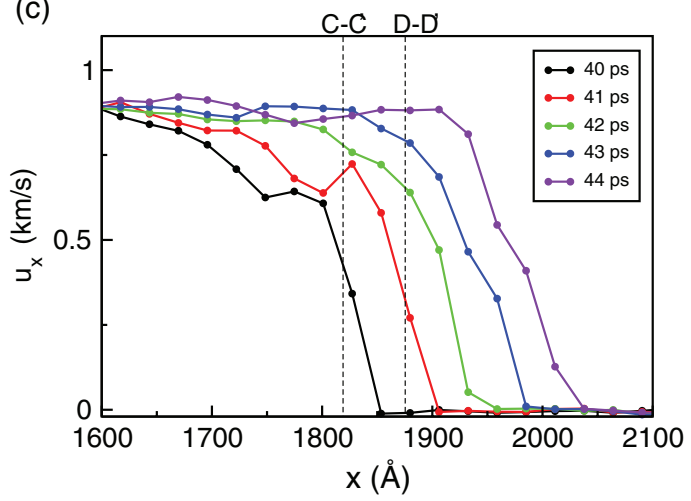

FIG. 13. (a) 2D distribution of temperature in regular hexagonal columnar nanocrystalline PETN, averaged over the $L$-direction for the $T 2$-direction loading with $u_{\mathrm{p}}=1.0 \mathrm{~km} / \mathrm{s}$ at $t=50 \mathrm{ps}\left(\theta=30^{\circ}\right)$. (b) and (c) $1 \mathrm{D}$ particle velocity profiles of the middle portion of the target [the region bound by black dashed lines in (a)]. The black dashed lines, $A A^{\prime}, B B^{\prime}, C C^{\prime}$, and $D D^{\prime}$, in (b) and (c) are noted in (a). different angular misorientations $(\theta)$, as well as irregularhexagon-shaped grains. We explore the anisotropy in its shock response with three different loading directions $(T 1, T 2$, and $L$ ). We first discuss nanocrystalline PETN with regularhexagon-shaped grains loaded along the $T 1$-direction.

Figure 6 shows typical 2D temperature distributions, $T(x, y)$, of nanocrystalline PETN with different angular misorientations, and $u_{\mathrm{p}}=1.0 \mathrm{~km} / \mathrm{s}$. Local high temperature zones or hotspots form mostly in the GB triple junction regions, and heat conduction raises the temperature in neighboring areas. The evolutions show that, as a shock wave progresses, hotspot temperature is highest at the shock front, and the hotspot features smear via heat conduction into grain interiors behind the shock. The hotspot characteristics vary with angular misorientations. For small $\theta\left(10^{\circ}-30^{\circ}\right)$, the hotspots are dominated by GB triple junctions and tilted GBs, but interior temperature becomes comparable to that at GBs for $40^{\circ}$. The interior temperature also depends on the orientation of individual grains, due to their own deformation, which may or may not be related to GBs. For instance, temperature in type-1 grains is considerably higher than in type- 2 and 3 grains in the case of $\theta=40^{\circ}$. With increasing $\theta$, the hotspot temperature and grain interior temperature after heat conduction both decrease.

At a higher shock strength $\left(u_{\mathrm{p}}=1.5 \mathrm{~km} / \mathrm{s}\right.$; Fig. 7), higher temperatures at GBs and within grain interiors are observed. However, different from the case of $u_{\mathrm{p}}=1.0 \mathrm{~km} / \mathrm{s}$, the temperature field is homogenized, and more importantly, pronounced hotspots form at GBs and GB triple junctions for large angular misorientations $\left(\theta=40^{\circ}\right)$. The increase in hotspot temperature due to increased shock strength is much less for smaller $\theta$ cases. The hotspot temperature is about $1300 \mathrm{~K}\left(\theta=20^{\circ}\right)$ and $1200 \mathrm{~K}\left(\theta=40^{\circ}\right)$ for $u_{\mathrm{p}}=1.0 \mathrm{~km} / \mathrm{s}$, and $2000 \mathrm{~K}\left(\theta=20^{\circ}\right)$ and $2800 \mathrm{~K}\left(\theta=40^{\circ}\right)$ for $u_{\mathrm{p}}=1.5 \mathrm{~km} / \mathrm{s}$. The temperature at GB hotspots is much higher than that within grain interiors, for example, it is $2800 \mathrm{~K}$ vs. $1200 \mathrm{~K}$ for $\theta=40^{\circ}$ and $u_{\mathrm{p}}=1.5$ km/s (Fig. 7).

A natural explanation for different hotspot features observed for different angular misorientations is the differences (a)

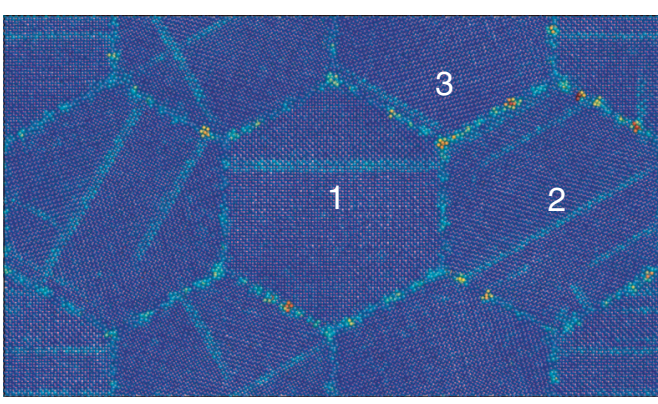

- [001] shock direction (b)

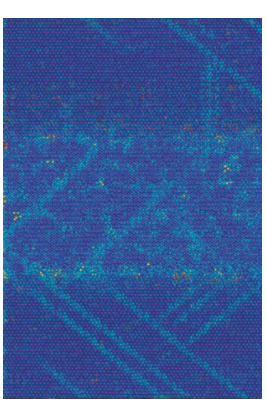

[001] shock direction

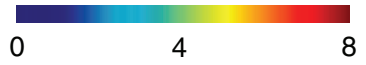

FIG. 14. 2D distribution of MRD for regular hexagonal columnar nanocrystalline PETN $\left(\theta=30^{\circ}\right)$ for the $L$-direction loading, viewed along the shock or [001] direction (a), and the $T 1$-direction (b). 

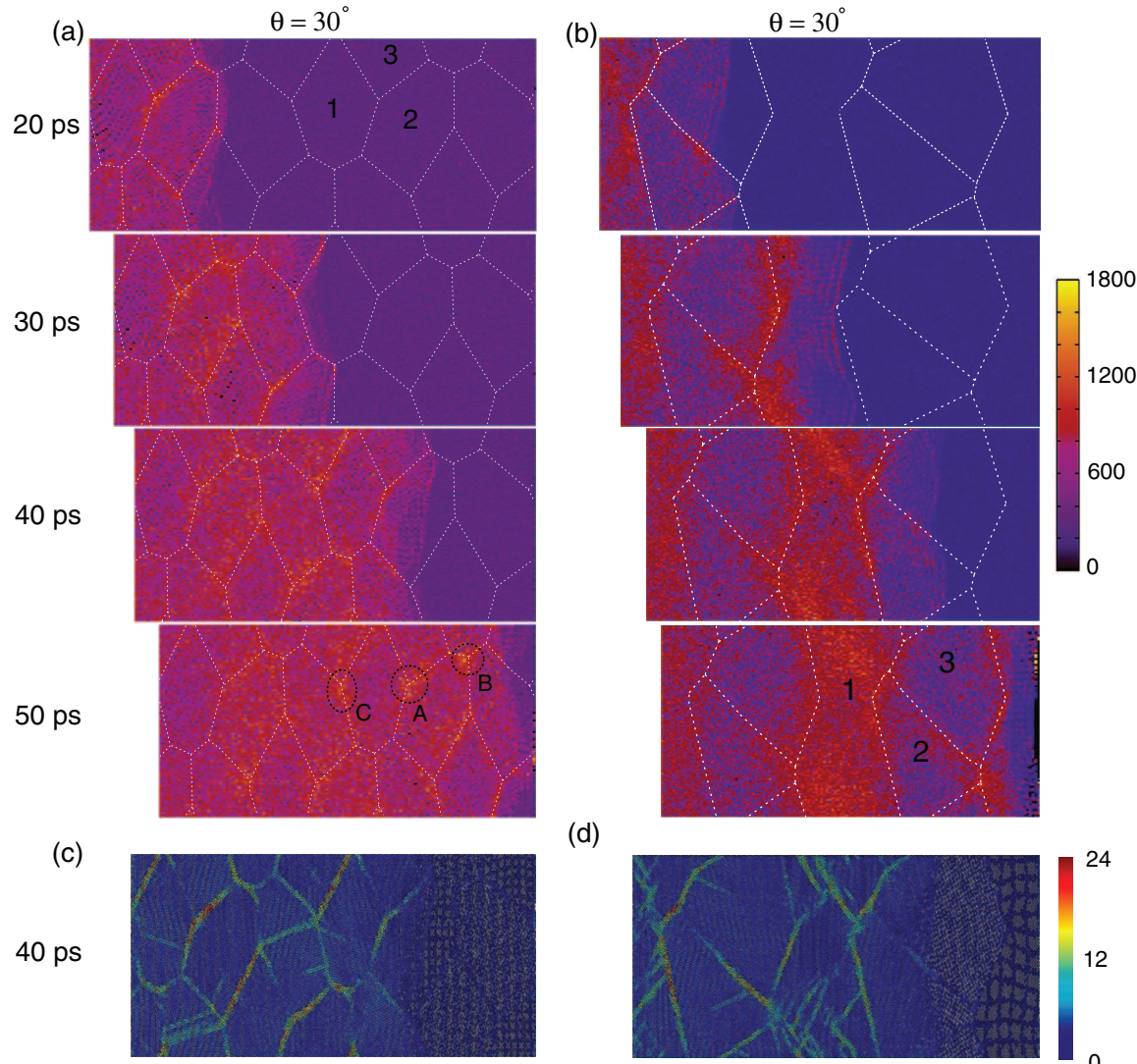

(d)

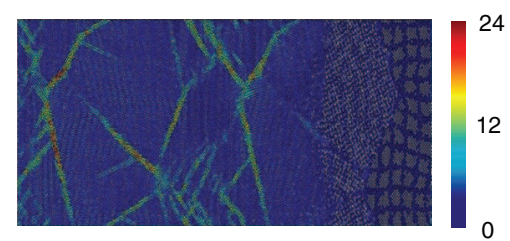

FIG. 15. Evolution of 2D distribution of temperature in irregular hexagonal (a) and random-shaped columnar (b) nanocrystalline PETN $\left(\theta=30^{\circ}\right)$, averaged over the $L$-direction, for $u_{\mathrm{p}}=1.0 \mathrm{~km} / \mathrm{s}$. The white dotted lines indicate the approximate locations of GBs. Shock direction: left $\rightarrow$ right. (c) and (d) are atomic configurations with MRD coding, for irregular-hexagonal and random-shaped nanocrystalline PETN, respectively.

in plastic deformation (Figs. 8-10), which in turn is due to crystal plasticity or GB deformation (the latter is referred to as "apparent" plasticity). One most important form of the GB

TABLE I. Resolved shear stresses (RSS) in single crystal PETN, for selected slip systems and shock planes. Calculations are done at $u_{\mathrm{p}}=0.3$ $\mathrm{km} / \mathrm{s}$. (12 50 ) and (4 30 ) shock planes refer to the single crystals rotated by $\theta=22^{\circ}$ and $\theta=37^{\circ}$ relative to the reference grain in Fig. 1(c), respectively.

\begin{tabular}{llcccc}
\hline \hline $\begin{array}{l}\text { Shock } \\
\text { plane }\end{array}$ & $\begin{array}{c}\text { Slip } \\
\text { system }\end{array}$ & $\begin{array}{c}\text { RSS } \\
(\mathrm{GPa})\end{array}$ & $\begin{array}{c}\text { Shock } \\
\text { plane }\end{array}$ & $\begin{array}{c}\text { Slip } \\
\text { system }\end{array}$ & $\begin{array}{c}\text { RSS } \\
(\mathrm{GPa})\end{array}$ \\
\hline$\left(\begin{array}{llllll}1 & 0 & 0\end{array}\right)$ & $(110)[1 \overline{1} 1]$ & 0.871 & $(1250)$ & $(110)[1 \overline{1} 0]$ & 0.285 \\
& $(101)[11 \overline{1}]^{\mathrm{a}}$ & 0.603 & & $(110)[1 \overline{1} \overline{1}]^{\mathrm{a}}$ & 0.249 \\
& $(110)[1 \overline{1} 0]^{\mathrm{a}}$ & 0.982 & & $(101)[11 \overline{1}]$ & 0.100 \\
& $(201)[\overline{1} 02]$ & 0.751 & & $(010)[101]$ & 0.280 \\
$\left(\begin{array}{llll}0 & 0 & 1\end{array}\right)$ & $(101)[11 \overline{1}]$ & 0.205 & & $(201)[10 \overline{2}]$ & 0.267 \\
& $(101)[\overline{1} 01]^{\mathrm{a}}$ & 0.268 & $(430)$ & $(110)[1 \overline{1} 0]$ & 0.116 \\
& $(201)[\overline{1} 02]$ & 0.284 & & $(110)[1 \overline{1} 1]$ & 0.105 \\
& $(110)[1 \overline{1} 1]$ & 0.001 & & $(101)[11 \overline{1}]$ & 0.084 \\
$\left(\begin{array}{llll}1 & 1 & 0\end{array}\right)$ & $(100)[0 \overline{1} \overline{1}]$ & 0.222 & & $(010)[10 \overline{1}]^{\mathrm{a}}$ & 0.238 \\
& $(101)[10 \overline{1}]$ & 0.175 & & $(201)[10 \overline{2}]$ & 0.226 \\
& $(201)[10 \overline{2}]$ & 0.172 & & $(101)[1 \overline{1} \overline{1}]^{\mathrm{a}}$ & 0.293 \\
& $(10 \overline{1})[1 \overline{1} 1]^{\mathrm{a}}$ & 0.250 & $(111)$ & $(001)[110]^{\mathrm{a}}$ & 0.952 \\
$\left(\begin{array}{llll}1 & 0 & 1\end{array}\right)$ & $(201)[\overline{1} 02]^{\mathrm{a}}$ & 0.484 & & $(110)[1 \overline{1} 1]$ & 0.460 \\
& $(001)[110]$ & 0.445 & & $(201)[\overline{1} 02]$ & 0.267 \\
& $(100)[011]$ & 0.529 & & $(101)[11 \overline{1}]$ & 0.588 \\
& $(110)[1 \overline{1} 1]$ & 0.239 & & $(100)[011]$ & 0.671 \\
\hline \hline
\end{tabular}

a Slip systems observed at higher shock strength for the current shock plane (the rest slip systems are for other shock planes). deformation is friction (Fig. 10), for its efficiency in inducing local heating. GB deformation may also be coupled to crystal plasticity since GBs are heterogeneous nucleation sites for dislocations and twins. Crystal plasticity induces local heating as well. Our simulations indicate that GB friction plays a key role in hotspot formation, and crystal plasticity initiated from GBs enhance local heating. As a shock passes by, GB friction may lead to GB-initiated crystal plasticity, but they may occur concurrently and be directly coupled for high shock strengths.

The plastic deformation at GBs and within grain interiors are shown as MRD distributions and MRD vector plot in Figs. 8-10. Crystal plasticity is manifested as dislocations and sometimes nanotwins (Figs. 8 and 9), mainly nucleated from the GBs, while homogeneous nucleation is evident within type-1 grains for $\theta=40^{\circ}$ (Fig. 9). The slip planes inside the grains are identified as $\{110\}$ planes. The activated slip systems form "deformation bands" (Fig. 8) or subgrain microstructure at higher shock strengths (Fig. 9). Regarding GB friction, the atoms near GBs may slip primarily on the GBs [Fig. 10(a)], but slip direction may also form an angle with the GBs [Figs. 10(b)-10(d)].

Deformation within individual grains, along with GBs, affects the temperature distributions. Table I shows that RSS of the slip systems $(110)\langle 1 \overline{1} 0\rangle$ and (110) $\langle 1 \overline{1} 1\rangle$ decreases in the order of (100), (12 50$)\left(\theta=22^{\circ}\right)$ and (4 30$)\left(\theta=37^{\circ}\right)$ shock planes, i.e., they are more difficult to activate as $\theta$ increases. The slip systems are the easiest to activate for type-1 grains, and become more difficult for type- 2 and 3 grains. For 
a given shock strength, there are fewer slip planes activated for $\theta=40^{\circ}$ than $\theta=10^{\circ}$ or $20^{\circ}$ (e.g., $u_{\mathrm{p}}=1.0 \mathrm{~km} / \mathrm{s}$, Fig. 8). At a higher shock strength of $u_{\mathrm{p}}=1.5 \mathrm{~km} / \mathrm{s}$, deformation at grain interiors and GBs increases and hotspots of the highest temperature form at GBs for $\theta=40^{\circ}$ (Fig. 7).

We thus can correlate plastic deformation with local temperature. For example, the larger deformation within grains lead to higher interior temperature. Since the slip systems within all types of grains are easier to activate for small $\theta$, the grain temperature field is more homogenous (Fig. 7). For $\theta=40^{\circ}$, the delayed activation of the slip systems in type-2 and 3 grains help the accumulation of strain energy, and its rapid release upon activation contributes to high temperature hotspot formation at $u_{\mathrm{p}}=1.5 \mathrm{~km} / \mathrm{s}$ (Fig. 7). These hotspots, are nonetheless a combined result of crystal plasticity and GB friction (see discussion below), i.e., the deformation in type2 and 3 grains, coupled with GB frictions, gives rise to the hotspots, although type-1 grains have undergone pronounced deformation even at $u_{\mathrm{p}}=1.0 \mathrm{~km} / \mathrm{s}$. This is consistent with the steric hindrance model for single crystals: although steric hindrance impedes or delays plastic deformation, it leads to energy accumulation, and then violent deformation and heat production at a higher load, yielding sufficiently high local temperature (hotspots) to initiate chemical reaction (high sensitivity). Therefore, sensitivity of single crystals is still relevant in polycrystalline sensitivity.

A high MRD magnitude at GBs indicates high deformation, and normally indicates high temperature. However, there are exceptions, since the slip direction has to be considered. Figure 9 shows three high-slip GB areas, and their magnitudes are in the order of $A>C>B$; however, the corresponding local temperatures are in the order of $B>A>C$ (Fig. 7). The major difference is that the slip direction coincides approximately with the GBs for $A$ and $C$, while there are appreciable out-of-plane components for $B$. Since GBs are expectedly less resistant to friction, the deviation of slip from the GB in the case of $B$ leads to higher friction and temperature. The lower magnitude and less deviation in slip direction from GB for the case of Fig. 10(d) results in a negligible increase in temperature [the arrowed GB, Fig. 6], highlighting the importance of slip direction of GB atoms in inducing frictional heating.

\section{Columnar nanocrystalline PETN: Other loading directions and irregular grain shapes}

We also simulate the shock response of PETN with regular hexagonal grains shocked along the $T 2$ - and $L$-directions, and nanocrystalline PETN with irregular hexagonal/random shaped grains and $\theta=30^{\circ}$, shocked along the $T 1$-direction.

Figure 11 shows typical temperature fields, $T(x, y)$, for the T2-direction loading of regular hexagonal grained PETN with different misorientation angles at $u_{\mathrm{p}}=1.0 \mathrm{~km} / \mathrm{s}$ and 1.5 $\mathrm{km} / \mathrm{s}$. The general features are well consistent with the results of the T1-direction loading. However, the spatial periodicity of hotspots is more evident for the T2-direction loading. As expected, the hotspot temperature is approximately the same as for $u_{\mathrm{p}}=1.0 \mathrm{~km} / \mathrm{s}$, while the hotspot temperature for large $\theta$ is much higher than that for small $\theta$ in the case of $u_{\mathrm{p}}=1.5$ $\mathrm{km} / \mathrm{s}$.
Similar to the T1-direction loading, the mechanisms of hotspot formation are still GB friction and GB-initiated crystal plasticity. Homogeneous nucleation of plasticity in grain interiors induces temperature increase, but this heating effect is much less efficient than GBs. The 2D distributions of MRD for the $T 2$-direction loading with $u_{\mathrm{p}}=1.0 \mathrm{~km} / \mathrm{s}$ and $1.5 \mathrm{~km} / \mathrm{s}$ are displayed in Fig. 12. Crystal plasticity is nucleated from GB triple junctions and GBs, and homogeneous nucleation occurs mostly within type-1 grains. The deformation near the GBs and within type- 2 and 3 grains decreases with increasing $\theta$, but the deformation in grain 1 increases. The decreasing deformation in type- 2 and 3 grains can be accounted for by the decrease in RSS with increasing $\theta$. In the absence of inelastic deformation in type- 2 and 3 grains which would relieve the shear stress imparted by the shock, strain is accommodated in type-1 grains via the activation of slip systems. When $u_{\mathrm{p}}$ reaches $1.5 \mathrm{~km} / \mathrm{s}$, homogenous nucleation of plasticity increases significantly. Besides crystal plasticity, GB friction, in particular, the deviation of slip direction away from GBs, is important in hotspot formation, as observed for the T1-direction loading.

While hotspot formation is directly related to GB friction and GB-initiated crystal plasticity, and can be explained with RSS and grain orientations, we examine below that wave propagation may also induce differences in local temperature rise, i.e., local rapid loading could contribute to local heating. When a shock wave propagates in a polycrystalline solid, the shock front structure may change dynamically as it traverses different local microstructure. As shown in Fig. 3, the separation between the elastic wave and plastic wave decreases with increasing $\theta$ [e.g., increasing $\theta$ in the order of (100), (12 5 $0),(430)$, and (110) shock planes]. As $\theta$ increases, the shock front thickness or equivalent shock rise width, $w$, decreases to nearly zero for the (110) shock plane with $\theta=45^{\circ}$. This single crystal behavior also manifests itself in wave propagation through a polycrystalline solid; the wave formed in a preceding crystal becomes the loading pulse on the following crystal.

The switch between single- and two-wave structures is illustrated with 1D particle velocity profiles (Fig. 13) along the shock direction in a region prescribed in Fig. 13(a). Figure 13(b) shows the single- to two-wave structure transition when the shock propagates from grains 3 and 2 to grain 1 . As the shock encounters $A A^{\prime}$ and $B B^{\prime}$, the single-wave shock front broadens and flattens (37 ps), and then a well-defined elastic precursor forms (after $38 \mathrm{ps).} \mathrm{An} \mathrm{opposite} \mathrm{case} \mathrm{is} \mathrm{pre-}$ sented in Fig. 13(c), where the two- to single-wave structure transition occurs when the shock propagates from grain 1 into grains 3 and 2 . When the two-wave shock in grain 1 reaches $C C^{\prime}$ and $D D^{\prime}$, the elastic precursor is suppressed, the particle velocity in the GB region of grains 2 and 3 rises gradually, subsequently forming a single-wave structure. In addition to shock front structure changes, there are corresponding changes in the peak particle velocity as the shock traverses grains with different orientations and thus different impedances.

The dynamic spatial changes in loading (e.g., the shock front structure and peak state) during its propagation can directly affect the spatial characteristics of physical 
properties in the region behind the front, including hotspots. As shown in Fig. 13(a), hotspots form within grains 2 and grain 3 in the GB triple junction regions $(O$ and $P$ ), and heat conduction also raises the temperature in the neighboring area. Conversely, the temperature in the right portion of the GB region in grain $1(Q$ and $R$ ) is relatively low when shock enters from grain 1 into grain 2 or 3 . This phenomenon is partly due to the steepness (or loading rate) of the shock front before it impinges on the GBs: it is much steeper for the path of grains 2 or $3 \rightarrow 1$ than that of grains $1 \rightarrow 2$ or 3 ; and another reason is that the peak values (e.g., $u_{\mathrm{p}}$ ) are also higher in grains 2 and 3 than in grain 1 . The strain concentrations at the GB triple junctions also contribute to elevated temperatures. The passage of a shock wave induces temperature gradients near GBs, and thermal diffusion continues to achieve thermal equilibrium.

Shock simulations along the $L$-direction of regular hexagonal columnar nanocrystalline PETN are also conducted. Figure 14 shows MRD for $u_{\mathrm{p}}=0.5 \mathrm{~km} / \mathrm{s}$ viewed from different directions. As Figure 4(c) shows, there is no inelastic deformation for the (001) shock plane loaded at $u_{\mathrm{p}}=0.5 \mathrm{~km} / \mathrm{s}$. However, the $\{101\}$ slip planes are activated in the nanocrystalline PETN, and the slip planes are mainly nucleated from the GBs. Since the loading is the same, including the shock direction ([001]) and loading pulse, for all three grains, the deformation in 3 types of grains is almost identical statistically. At increased shock strengths, high deformation is induced. However, the hotspot formation at GBs or within grain interiors is not marked, since the structure is essentially homogeneous along the shock direction, which lies on the GBs. As a result, stress concentrations, GB friction, and GB-enhanced deformation are fairly limited.

Since grain shapes may affect local wave field and stress/strain concentrations, we examine the shock responses of nanocrystalline PETN with irregular hexagonal [Figs. 15(a) and 15(c)] or random-shaped grains [Figs. 15(b) and 15(d)]. The latter configuration contains a type of grains of a concave polygon shape. Figure 15 shows the $2 \mathrm{D}$ temperature distributions and MRD maps for $u_{\mathrm{p}}=1.0 \mathrm{~km} / \mathrm{s}$. The bulk temperature is approximately the same for regular and irregular hexagonal columnar nanocrystalline PETN; however, the spatial distribution of hotspots is different. Hotspots form not only in the areas expected for regular hexagonal nanocrystalline PETN [A and $B$ in Fig. 15(a)], but also in the new areas $(C)$. The slip planes revealed by MRD are consistent with the regular hexagon case, indicating that the slip systems activated are not sensitive to GB shapes. Figure 15(b) shows the case of random GBs. Its spatial distribution of hotspots differs greatly from the regular/irregular hexagon cases, with strong bias towards grain $1 \mathrm{GBs}$. The temperature in grain 1 is much higher than that in grains 2 and 3 . The stress concentration near the concave edges of the grain account for these observations. In addition, RSS in grain 1 also favors larger deformation than in grains 2 and 3 . However, the slip systems activated stay the same. The deformation features shown in Figs. 15(c) and $15(\mathrm{~d})$ are consistent with temperature distributions [Figs. 15(a) and 15(b)].

Our simulations show that high temperature hotspots can be produced at GBs during shock loading, e.g., 2800 K for regular hexagonal nanocrystalline PETN with $\theta=40^{\circ}$ loaded at $u_{\mathrm{p}}=1.5 \mathrm{~km} / \mathrm{s}$. However, some questions remain open, including the critical temperature at elevated pressures, critical hotspot volume, and critical time scale of sustained temperature (before heat conduction reduces it). In particular, direct, large-scale, ReaxFF simulations of GB and other microstructure effects are highly desirable. We also need to point out that a limitation of the $\mathrm{CG}$ model is its reduced number of degrees of freedom in the molecules. As a result, the exact values of temperatures may be affected. ${ }^{23}$ Thus, the temperature values should not be taken literally, and rather represent a trend in a semi-quantitative way. A direct comparison of all-atom simulations with some of the CG-MD simulations will be done in the future. While our simulations indeed demonstrate the possible role of GBs in hotspot formation, the limitations should be considered, such as the small grain sizes in our simulations, the complexities of microstructure in real systems, and competing hotspot mechanisms such as void collapse.

\section{CONCLUSIONS}

The shocked single crystals show pronounced anisotropy in elastic and plastic deformation. Plastic deformation, slip systems, and resolved shear stresses are characterized. RSS is higher for (110) and (001) shock planes than the (100), (101), and (111) cases, and shock-induced plasticity is consistent with RSS calculations and the steric hindrance model. Plasticity-induced local heating (mechanical hotspots) is expected to lead to chemical hotspots for initiation.

Shock responses of columnar nanocrystalline PETN with regular and irregular hexagonal grains, and with random grain shapes, demonstrate strong anisotropy and varying hotspot characteristics, as well as strong dependence on the angular misorientations. Within the context of microstructure with GBs only, some key points are

- Mechanical hotspot formation is directly related to GB friction and GB-initiated crystal plasticity, and the exact deformation is dictated by grain orientations and resolved shear stress. While GB friction alone can induce mechanical hotspots, the hotspot temperature can be greatly enhanced if coupled with GB-initiated crystal plasticity.

- The magnitude of shearing in terms of MRD amplitude, can correlate well with temperature. This is true for plasticity within grain interiors. However, exceptions do exist for GBs deformation, since smaller slip off the GBs may be much more efficient in friction heating than larger slip on GBs. The slip directions of GB atoms are critical to the efficiency of frictional heating.

- Stress concentrations occur due to heterogeneous structures and drive the deformation, and depend on GB characteristics such as shape and orientation. Wave propagation through varying microstructure may also induce differences in stress states and loading rates, and thus, local temperature rise (e.g., rapid local loading can induce more heating). 
- GBs are much more efficient in nucleating mechanical hotspots than single crystal plasticity, and the effective volume of materials and temperature are considerable higher.

\section{ACKNOWLEDGMENTS}

This work was supported in part by National Science Foundation of China (NSFC) (11172289) and by the Fundamental Research Funds for the Central Universities of China. Q.A. and W.A.G. acknowledge the support by (U.S.) Army Research Office (USARO) (W911NF-05-1-0345 and W911NF-08-1-0124) and by Office of Naval Research (ONR) (N00014-09-1-0634).

${ }^{1}$ F. P. Bowden and Y. D. Yoffe, Initiation and Growth of Explosion in Liquids and Solids (Cambridge University Press, Cambridge, 1952).

${ }^{2}$ J. P. Hirth and J. Lothe, Theory of Dislocations (John Wiley and Sons, New York, 1982).

${ }^{3}$ D. Hull and D. J. Bacon, Introduction to Dislocations, 3rd ed. (Pergamon Press, New York, 1984)

${ }^{4}$ R. W. Armstrong, Rev. Adv. Mater. Sci. 19, 13 (2009).

${ }^{5}$ H. W. Sandusky, B. C. Glancy, D. W. Carlson, W. L. Elban, and R. W. Armstrong, J. Propul. Power 7, 518 (1991).

${ }^{6}$ B. L. Holian, T. C. Germann, J. B. Maillet, and C. T. White, Phys. Rev. Lett. 89, 285501 (2002).

${ }^{7}$ L. Tran and H. S. Udaykumar, J. Propul. Power 22, 947 (2006).

${ }^{8}$ L. Tran and H. S. Udaykumar, J. Propul. Power 22, 959 (2006)

${ }^{9}$ R. Menikoff, Combust. Theory Modell. 10, 1003 (2006).

${ }^{10}$ Y. Hu, D. W. Brenner, and Y. Shi, J. Phys. Chem. C 115, 2416 (2011).

${ }^{11}$ W. Slough and W. F. Perger, Chem. Phys. Lett. 498, 97 (2010).

${ }^{12}$ E. F. C. Byrd and B. M. Rice, J. Phys. Chem. C 111, 2787 (2007).

${ }^{13}$ M. M. Budzevich, A. C. Landerville, M. W. Conroy, Y. Lin, I. I. Oleynik, and C. T. White, J. Appl. Phys. 107, 113524 (2010).

${ }^{14}$ W. F. Perger, S. Vutukuri, Z. A. Dreger, Y. M. Gupta, and K. Flurchick, Chem. Phys. Lett. 422, 397 (2006).

${ }^{15}$ N. Desbiens, E. Bourasseau, and J. B. Maillet, Mol. Simul. 33, 1061 (2007).

${ }^{16}$ L. He, T. D. Sewell, and D. L. Thompson, J. Chem. Phys. 134, 124506 (2011).

${ }^{17}$ S. D. Herring, T. C. Germann, and N. Gronbech-Jensen, Phys. Rev. B 82, $214108(2010)$

${ }^{18}$ Q. An, S. V. Zybin, W. A. Goddard III, A. Jaramillo-Botero, M. Blanco, and S. N. Luo, Phys. Rev. B 84, 220101(R) (2011).

${ }^{19}$ J. Budzien, A. P. Thompson, and S. V. Zybin, J. Phys. Chem. B 113, 13142 (2009)

${ }^{20}$ R. H. Gee, C. Wu, and A. Maiti, Appl. Phys. Lett. 89, 021919 (2006).

${ }^{21}$ K. Lynch, A. Thompson, and A. Strachan, Modell. Simul. Mater. Sci. Eng. 17, 015007 (2009)

${ }^{22} \mathrm{~S}$. Izvekov, P. W. Chung, and B. M. Rice, The Multiscale CoarseGraining Method: Assessing its Accuracy and Introducing Density Dependent Coarse-Grain Potentials (AIP, New York, 2010), Vol. 133.

${ }^{23}$ S. Izvekov, P. W. Chung, and B. M. Rice, J. Chem. Phys. 135, 044112 (2011)

${ }^{24}$ D. C. Sorescu, B. M. Rice, and D. L. Thompson, J. Phys. Chem. B 104, 8406 (2000).

${ }^{25}$ P. M. Agrawal, B. M. Rice, and D. L. Thompson, J. Chem. Phys. 119, 9617 (2003)

${ }^{26}$ L. Q. Zheng, S. N. Luo, and D. L. Thompson, J. Chem. Phys. 124, 154504 (2006).

${ }^{27}$ A. Hervouët, N. Desbiens, E. Bourasseau, and J. B. Maillet, J. Phys. Chem. B 112, 5070 (2008).
${ }^{28}$ N. Desbiens, E. Bourasseau, J. B. Maillet, and L. Soulard, J. Hazard. Mater. 166, 1120 (2009).

${ }^{29}$ A. Siavosh-Haghighi, R. Dawes, T. D. Sewell, and D. L. Thompson, J. Chem. Phys. 131, 064503 (2009).

${ }^{30}$ G. D. Smith and R. K. Bharadwaj, J. Phys. Chem. B 103, 3570 (1999).

${ }^{31}$ D. Bedrov, G. D. Smith, and T. D. Sewell, Chem. Phys. Lett. 324, 64 (2000).

${ }^{32}$ D. Bedrov, G. D. Smith, and T. D. Sewell, J. Chem. Phys. 112, 7203 (2000).

${ }^{33}$ T. D. Sewell, R. Menikoff, D. Bedrov, and G. D. Smith, J. Chem. Phys. 119, 7417 (2003).

${ }^{34}$ E. Jaramillo, T. D. Sewell, and A. Strachan, Phys. Rev. B 76, 064112 (2007).

${ }^{35}$ A. Strachan, A. C. T. van Duin, D. Chakraborty, S. Dasgupta, and W. A. Goddard III, Phys. Rev. Lett. 91, 098301 (2003).

${ }^{36}$ A. Strachan, E. M. Kober, A. C. T. van Duin, J. Oxgaard, and W. A. Goddard, J. Chem. Phys. 122, 054502 (2005).

${ }^{37}$ M. J. Cawkwell, T. D. Sewell, L. Q. Zheng, and D. L. Thompson, Phys. Rev. B 78, 014107 (2008).

${ }^{38}$ D. Bedrov, J. B. Hooper, G. D. Smith, and T. D. Sewell, J. Chem. Phys. 131, 034712 (2009).

${ }^{39}$ M. J. Cawkwell, K. J. Ramos, D. E. Hooks, and T. D. Sewell, J. Appl. Phys. 107, 063512 (2010).

${ }^{40}$ K. J. Ramos, D. E. Hooks, T. D. Sewell, and M. J. Cawkwell, J. Appl. Phys. 108, 066105 (2010).

${ }^{41}$ L. B. Munday, P. W. Chung, B. M. Rice, and S. D. Solares, J. Phys. Chem. B 115, 4378 (2011).

${ }^{42}$ N. Mathew and R. C. Picu, J. Chem. Phys. 135, 024510 (2011).

${ }^{43}$ B. M. Rice and T. D. Sewell, in Static Compression of Energetic Materials, 1st ed., edited by S. M. Peiris and G. J. Piermarini (Springer-Verlag, New York, 2008), p. 330.

44 J. J. Dick and J. P. Ritchie, J. Appl. Phys. 76, 2726 (1994).

${ }^{45}$ J. J. Dick, Appl. Phys. Lett. 44, 859 (1984).

${ }^{46}$ J. J. Dick, R. N. Mulford, W. J. Spencer, D. R. Pettit, E. Garcia, and D. C. Shaw, J. Appl. Phys. 70, 3572 (1991).

${ }^{47}$ J. J. Dick, J. Appl. Phys. 81, 601 (1997)

${ }^{48}$ J. M. Winey and Y. M. Gupta, J. Appl. Phys. 107, 103505 (2010).

${ }^{49}$ R. M. Eason and T. D. Sewell, J. Phys. Chem. C 116, 2226 (2012).

${ }^{50}$ S. V. Zybin, W. A. Goddard, P. Xu, A. C. T. van Duin, and A. P. Thompson, Appl. Phys. Lett. 96, 081918 (2010).

${ }^{51}$ B. Arman, A. S. Reddy, and G. Arya, Macromolecules 45, 3247 (2012).

${ }^{52}$ S. Plimpton, J. Comput. Phys. 117, 1 (1995). Also see http://lammps. sandia.gov.

${ }^{53}$ A. Okabe, B. Boots, K. Sugihara, and S. N. Chiu, Concepts and Applications of Voronoi Diagrams, 2nd ed. (Wiley, Chichester, 2000).

${ }^{54}$ S. N. Luo, Q. An, T. C. Germann, and L. B. Han, J. Appl. Phys. 106, 013502 (2009).

${ }^{55}$ S. N. Luo, T. C. Germann, and D. L. Tonks, J. Appl. Phys. 107, 123507 (2010).

${ }^{56}$ B. Arman, Q. An, S. N. Luo, T. G. Desai, D. L. Tonks, T. Cagin, and W. A. Goddard III, J. Appl. Phys. 109, 013503 (2011).

${ }^{57}$ J. A. Zimmerman, C. L. Kelchner, P. A. Klein, J. C. Hamilton, and S. M. Foiles, Phys. Rev. Lett. 87, 165507 (2001).

${ }^{58}$ Q. An, Y. Liu, S. V. Zybin, H. Kim, and W. A. Goddard III, J. Phys. Chem. C 116, 10198 (2012)

${ }^{59}$ A. V. Bushman, I. V. Lomonosov, K. V. Khishchenko, V. E. Kogan, P. R. Levashov, I. N. Lomov, and P. R. Levashov, Shock Wave Database, the online version at http://www.ficp.ac.ru/rusbank/index.php, and references therein

${ }^{60}$ S. P. Marsh, LASL Shock Hugoniot Data (University of California Press, Berkeley, 1980).

${ }^{61}$ P. M. Halleck and J. Wackerle, J. Appl. Phys. 47, 976 (1976).

${ }^{62}$ T. C. Germann, B. L. Holian, P. S. Lomdahl, and R. Ravelo, Phys. Rev. Lett. 84, 5351 (2000).

${ }^{63}$ R. Ravelo, Q. An, T. C. Germann, and B. L. Hollian, AIP Conf. Proc. 1426, 1263 (2011) 